\title{
Graphene oxide-upconversion nanoparticle based optical sensors for targeted detection of mRNA biomarkers present in Alzheimer's disease and prostate cancer
}

\author{
Patrick Vilela, ${ }^{a}$ Afaf El-Sagheer, ${ }^{\text {c,d }}$ Timothy M. Millar, ${ }^{e}$ Tom Brown, ${ }^{c}$ Otto L. Muskens, ${ }^{\text {a,b }}$ and \\ Antonios G. Kanaras ${ }^{a, b, *}$
}

${ }^{a}$ Physics and Astronomy, Faculty of Physical Sciences and Engineering, ${ }^{b}$ Institute of Life Sciences, University of Southampton, Southampton, SO171BJ, UK, ${ }^{\mathrm{c}}$ Department of Chemistry, University of Oxford, Chemistry Research Laboratory, 12 Mansfield Road, Oxford, OX1 3TA, UK, ${ }^{\mathrm{d}}$ Chemistry Branch, Department of Science and Mathematics, Faculty of Petroleum and Mining Engineering, Suez University, Suez 43721, Egypt, ${ }^{\mathrm{e}}$ Faculty of Medicine, University of Southampton, Southampton, SO16 6YD, UK.

*E-mail: A.Kanaras@ soton.ac.uk

\begin{abstract}
The development of new sensors for the accurate detection of biomarkers in biological fluids is of ultmost importance for the early diagnosis of diseases. Next to advanced laboratory techniques, there is a need for relatively simple methods which can significantly broaden the availability of diagnostic capability. Here, we demonstrate the successful application of a sensor platform based on graphene oxide and upconversion nanoparticles (NPs) for the specific detection of mRNA-related oligonucleotide markers in complex biological fluids. The combination of near-infrared light upconversion with low-background photon counting readout enables reliable detection of low quantities of small oligonucleotide sequences in the femtomolar range. We demonstrate the successful detection of analytes relevant to mRNAs present in Alzheimer's disease as well as prostate cancer in human blood serum. The high performance and relative simplicity of the upconversion NPgraphene sensor platform enables new opportunities in early diagnosis based on specific detection of oligonucleotide sequences in complex environments.
\end{abstract}

Keywords: upconversion nanoparticles, DNA, graphene oxide, biomarkers, biosensor, mRNA

Messenger RNAs (mRNAs) are biomolecules, which convey the necessary information for the ribosomal synthesis of proteins. ${ }^{1}$ The detection of intracellular mRNA levels is of high importance in the monitoring of the progression of disease ${ }^{2-4}$, development of targeted drug treatments ${ }^{5,6}$ and onset diagnosis of disorders ${ }^{7-9}$. Several methods are available to detect the presence of specific mRNAs, such as Northern blotting, RNase Protection Assay, DNA microarrays and in situ hybridization. ${ }^{10-13}$ For more sensitive studies of mRNA expression, real-time reverse-transcription quantitative pol- ymerase chain reaction ( $\mathrm{RT}-\mathrm{qPCR}$ ) is considered the standard protocol capable of quantifying RNA content from a volume as small as a single cell. ${ }^{14}$ While these techniques are powerful tools for the research laboratory, the level of technical skill and specialist equipment required in preparation and analysis protocols often poses a disadvantage, limiting the widespread availability of such methods. Recently, a variety of sensors have been developed which are based on the Förster resonance energy transfer (FRET) effect. ${ }^{15-20}$ The sensitivity of these sensors is highly dependent on the efficiency of the fluorophore donor. Commonly used dyes have several drawbacks, including photo-bleaching, blinking effects and interferences from biomolecules in samples that absorb in the UV-Vis range, usually overlapping with the absorption regions of most common dyes. ${ }^{21}$ To overcome these issues, upconversion nanoparticles (UCNPs) have been developed as an alternative fluorescence donor. UCNPs are lanthanide-doped nanoparticles, which are able to absorb two or more low energy photons and emit fluorescence at a shorter wavelength than the excitation wavelength. Typically, UCNPs absorb in the near-infrared (NIR) region and emit in the visible region of the electromagnetic spectrum. ${ }^{22}$ Additionally, UCNPs have great chemical and photochemical stability, low toxicity and an absence of photo-bleaching or blinking effects. ${ }^{23-26}$ When considering possible candidates for energy transfer acceptors, graphene oxide (GO) appears to be ideal, due to its high solubility in water ${ }^{25}$, high surface area and efficient quenching ${ }^{27,28}$ and its characteristic affinity to various biomolecules. ${ }^{29}$ For example, single stranded oligonucleotides are preferentially adsorbed to GO via $\pi-\pi$ stacking interactions, whereas double stranded oligonucleotides do not bind. ${ }^{30}$ Recently, our group developed a sensor based on graphene oxide and upconversion nanoparticles to detect short poly-A sequences with the detection limit in the picomolar range (see SI Scheme S1). ${ }^{31}$ Here, we show for the first time the applicability of upconversion nanoparticle/graphene oxide sensors for the detection of mRNA-related biomarkers. We have selected the mRNA biomarker BACE-1 related to Alzheimer's disease ${ }^{32}$ and the mRNA biomarker PCA3 related to prostate cancer ${ }^{33-35}$ to demonstrate the applicability of the technique relevant for early de- 


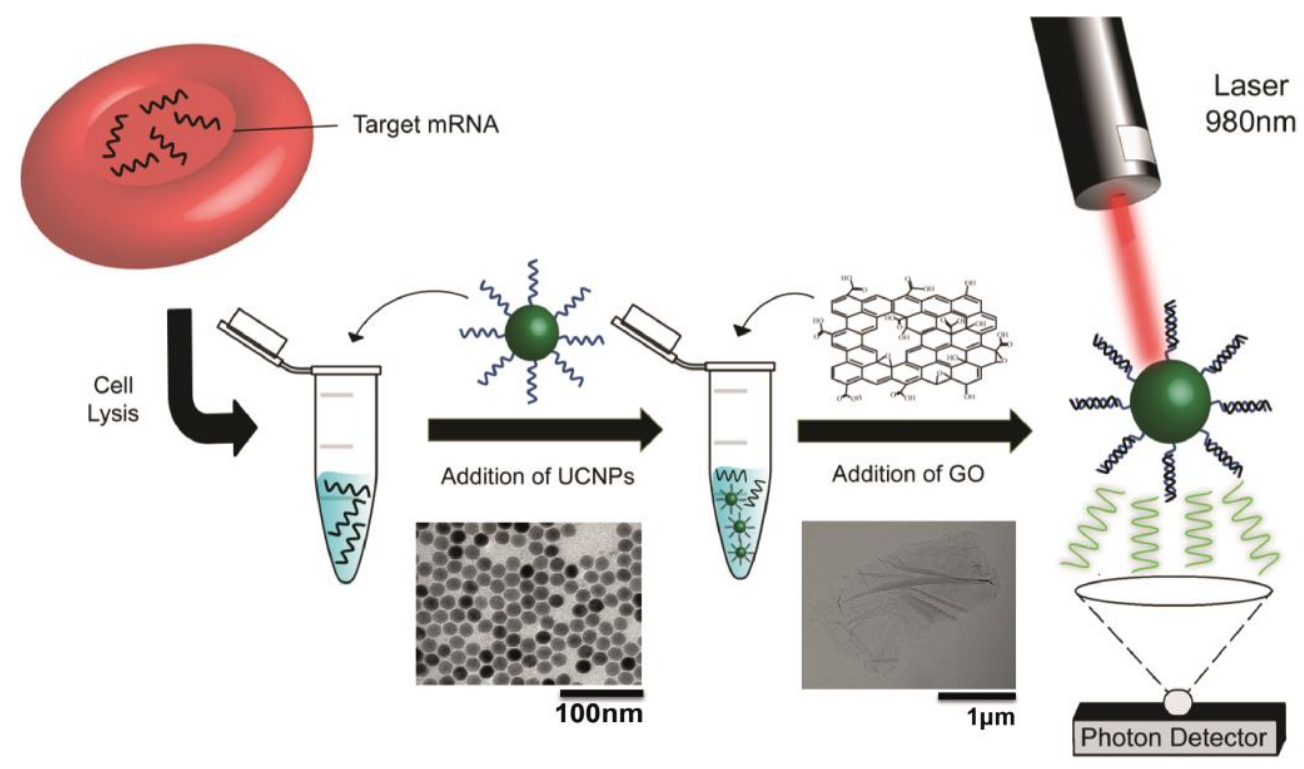

Scheme 1. Detection of biomarkers present in biopsy tissue or harvested cells. After lysis of the cells or tissue to be analyzed, the lysate is mixed firstly with the UCNPs (TEM image) coated with a sequence complementary to the target of interest. Then, the graphene oxide (GO, TEM image) solution is added and the sample is irradiated with a laser at $980 \mathrm{~nm}$. Monitoring of the fluorescent signature of the particles confirms the presence of the target sequence or not.

tection of critical diseases. By using a single-photon counting detection scheme, we achieve a detection sensitivity of target oligonucleotide sequences down to the femtomolar range. Most importantly our sensors retain their sensitivity and selectivity in complex biological fluids such as blood plasma and blood-cell lysate. A schematic illustration of the function of the sensors is shown in Scheme 1. A complex environment such as whole cell lysate is used as the starting solution to which UCNPs and GO are subsequently added in a two-step process. In the presence of BACE-1 or PCA3 target sequences, hybridization of the nucleotide chain results in a reduced adsorption of the UCNPs to the GO, which is subsequently detected by the fluorescent signature of the upconversion nanoparticles mediated by a $980 \mathrm{~nm}$ laser.

\section{Results and Discussion}

Synthesis and functionalization of upconversion nanoparticles The synthesis of NaYF4:Yb,Er nanoparticles was achieved following a previously established procedure ${ }^{36,37}$ and characterized via different microscopy and spectroscopy techniques (see SI for all the characterization). The particles were monodisperse with a mean diameter of $27 \pm 2 \mathrm{~nm}$ (see TEM image analysis in SI Figures S1A and S1B). The synthesized particles had a characteristic fluorescence signature relevant to their composition (SI Figures S1C and $\mathrm{S} 1 \mathrm{D})$. Nanoparticles were coated with oleic acid (OA) and dissolved in non-polar solvents. In order to transfer these particles into water, a ligand exchange reaction with polyacrylic acid (PAA) was performed following a simple substitution method. ${ }^{38}$ The advantages of this method are that the particles can easily transfer into water and that the ligand shell contains several carboxylic groups that can be further reacted via coupling reactions (SI Scheme S2). For the purpose of our experiments, amine-functionalized oligonucleotides were attached to the carboxylic groups of the nanoparticles via EDC/sulfo-NHS coupling. Three different oligonucleotide strands were employed. Two sense strands were designed to target selected areas of mRNAs associated with Alzheimer disease
(BACE-1) or prostate cancer (PCA3) (SI Table S1). A third, nonspecific strand containing a poly-T sequence was designed as a general sequence that can detect the poly-A tail of any mRNA. The covalent binding of the oligonucleotides to the nanoparticle polymer shell was confirmed with $\zeta$-Potential,UV-vis spectroscopy and FT-IR. Upon attachment of the sense strands to the nanoparticles, the zeta potential changed to a more negative value for all three types of oligonucleotides. This shift indicated the binding of the highly negative oligonucleotide strands to the nanoparticles (see ESI Figure S2A). The presence of oligonucleotides on the nanoparticle's surface was also confirmed with the appearance of a $280 \mathrm{~nm}$ peak on the UV spectra (SI Figure S2B). Finally, the successful EDC/sulfo-NHS attachment was confirmed via FT-IR (see SI Figure S2C), where a shift from $\sim 1640 \mathrm{~cm}^{-1}$ to a double peak at $\sim 1580 / \sim 1675 \mathrm{~cm}^{-1}$ confirmed the formation of the amide bond. The resulting oligonucleotide coated UCNPs were very stable and showed the characteristic Er-upconversion fluorescence spectrum under excitation with light from a $980 \mathrm{~nm}$ wavelength laser source (SI Figure S1C).

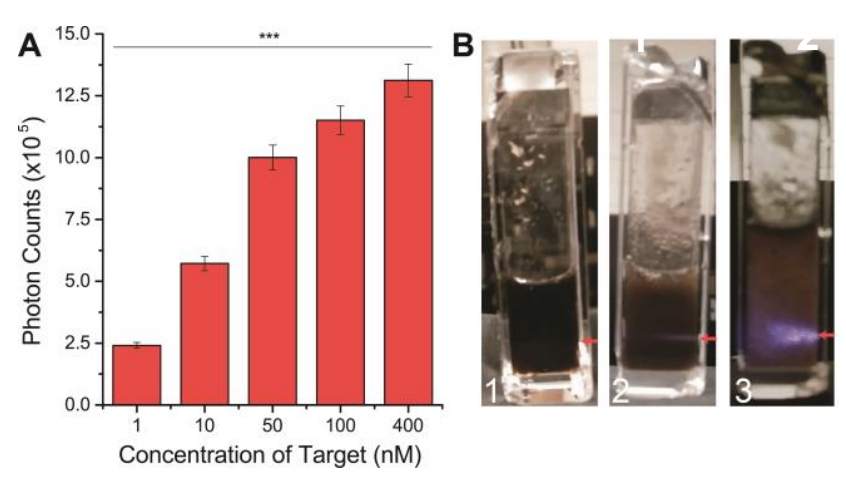

Figure 1. a) Detection of $545 \mathrm{~nm}$ emitted photons from oligonucleotide coated UCNPs $\left(0.5 \mathrm{mg} \cdot \mathrm{mL}^{-1}\right)$ in the presence of $\mathrm{GO}\left(0.5 \mathrm{mg} \cdot \mathrm{mL}^{-1}\right)$ and different concentrations of the target sequence poly-A $(* * * \mathrm{p}<0.001)$. The detection of the target is directly propor- 
tional to the amount of target present in solution. b) Digital photographs of the oligonucleotide coated UCNPs $\left(0.5 \mathrm{mg} \cdot \mathrm{mL}^{-1}\right)$ mixed with $\mathrm{GO}\left(0.5 \mathrm{mg} \cdot \mathrm{mL}^{-1}\right)$ in the absence of any target (1) and in the presence of $10 \mathrm{nM}(2)$ and $400 \mathrm{nM}$ (1) of poly-A target. Upconversion is visible as faint glow in (2) and (3) around the incident NIR laser beam (indicated by the red arrows).

\section{Targeting specificity and calculation of the detection limit}

Calibration of the sensors was achieved by adding various concentrations of graphene oxide ranging from 0 to $1 \mathrm{mg} \cdot \mathrm{mL}^{-1}$ to a fixed concentration of oligonucleotide coated upconversion nanoparticles $\left(0.5 \mathrm{mg} \cdot \mathrm{mL}^{-1}\right)$. The photon counts decreased proportionally with increasing concentrations of GO (see SI Figure S3). The lowest concentration of GO that achieves the highest degree of quenching was determined to be $0.5 \mathrm{mg} \cdot \mathrm{mL}^{-1}$. To test the stability of the sensor we monitored its performance over a period of 30 days (SI Figure S4). There were no significant variations in the values obtained, demonstrating the high stability of the sensor. Three different batches of oligonucleotide coated UCNPs were synthesized using sequences for the detection of Poly-A tails, BACE-1 and PCA3 (SI Table S1). Each batch was incubated with the respective target sequence. Figure 2 shows the detection of the targets over a range of concentrations from $200 \mathrm{fM}$ to $5 \mathrm{nM}$. The detection limit for every target was directly proportional to the concentrations of the respective sequence. The lowest experimental detection limit for poly-A was $200 \mathrm{fM}$ whereas BACE-1 and PCA3 sequences were detected at $500 \mathrm{fM}$ (SI Figure S5), since those concentrations were significantly above the detection limit threshold.

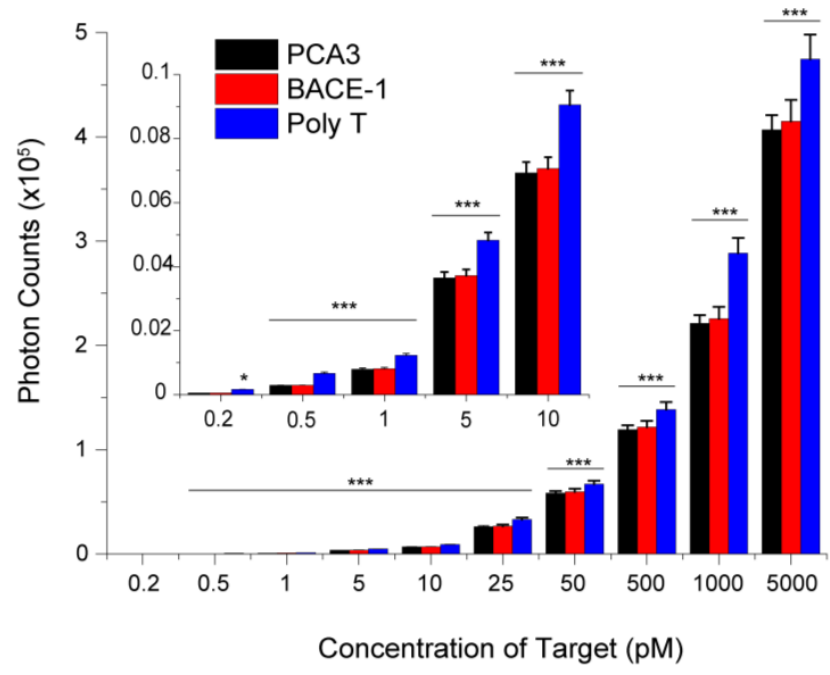

Figure 2. Performance of the sensors for the three oligonucleotide sense sequences (PCA3 (black), BACE-1 (red) and poly-T (blue) in PBS buffer ( $\left.{ }^{*} \mathrm{p}<0.01 ; * * * \mathrm{p}<0.001\right)$. An inset shows a magnification of the target detection for the range of $0.2-10 \mathrm{pM}$.

\section{Selectivity of the sensor and function in blood plasma and cell lysis}

An important aspect of a sensor is its ability to selectively detect a target in challenging environments, where biomolecules such as proteins, lipids and non-specific mRNAs could interfere with the detection of the target mRNA biomarkers.
The selectivity of our sensors was tested by subjecting them to both BACE-1 and PCA3 target oligonucleotide sequences at the same time. Figure 3A shows photon counts for BACE-1 (red) and PCA3 (black) sensors, when exposed to these oligonucleotide sequences. The results show the sensors to be highly selective for their respective target with minimal interference from the alternate oligonucleotide.

Subsequently, we tested the sensors both in whole blood cell lysate and in blood plasma, where the specific target was either present or absent. Blood was isolated from healthy volunteers and plasma and lysates were prepared using protocols described in SI Section VII. Figure 3B shows the performance of the sensors in both plasma and cell lysis media. In the presence of the target, all three sensors yielded a strong response comparable to that of the test solution of Figure 2. In absence of the target sequence, both the Alzheimer and prostate cancer sensors did not show a detection response either in plasma or cell lysate. In comparison, the poly-T oligonucleotide sequence sensor gave a strong fluorescent signal also in the absence of the poly-A sequence in the case of cell lysate. This observation is attributed to the presence of multiple mRNAs found in the cell lysate that carry a poly-A tail, which is detectable by the poly- $\mathrm{T}$ sensor, highlighting the excellent performance of the sensor for general mRNA targeting.
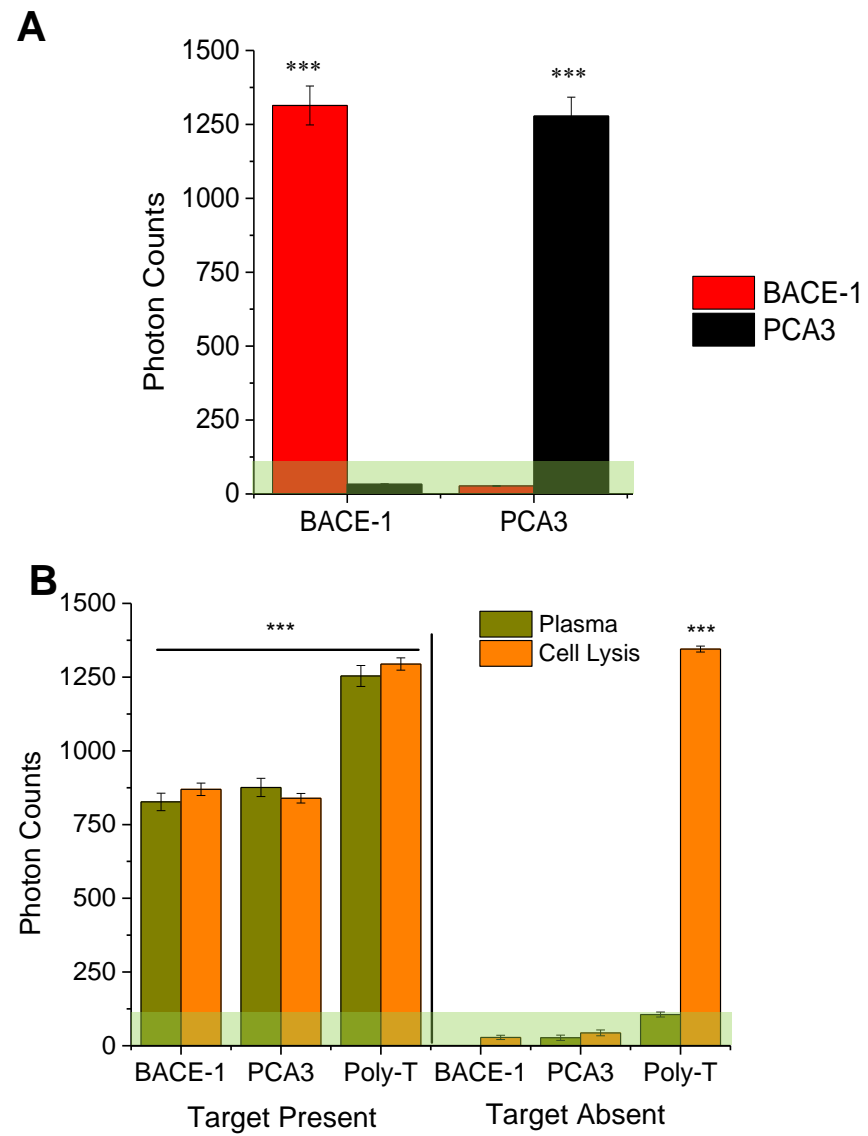

Figure 3. A) Specific oligonucleotide detection by the sensors in a cocktail of both BACE-1 and PCA3 targets at $1 \mathrm{pM}(* * * \mathrm{p}<0.001)$ in PBS. B) Performance of the sensors in the presence $(1 \mathrm{pM})$ and absence of the relevant oligonucleotide targets in plasma (dark green) and cell lysis (orange). In the absence of the target, the poly- $\mathrm{T}$ sensor is able to detect the poly-A tails of all mRNAs in cell lysis $(* * * \mathrm{p}<0.001)$. The noise limit is represented by the light green area. 


\section{Conclusions}

In summary, we successfully designed graphene oxide/upconversion nanoparticle sensors capable of detecting oligonucleotide sequences relevant to mRNA associated with Alzheimer's disease and prostate cancer. Our sensors have several important advantages when compared with previously reported systems. They are highly sensitive with a detection limit in the femtomolar range and with no drawbacks related to photo-blinking or biomolecule absorbance interferences. Furthermore, we have demonstrated that the sensor platform is highly selective to a specific target even in complex media such as blood plasma and blood cell lysate where the interference from other biomolecules can be significant. These sensors are specific, sensitive and adaptable for the detection of mRNA biomarkers, thus they can facilitate the early diagnosis of critical diseases such as Alzheimer's disease and prostate cancer.

\section{Supporting Information}

The Supporting Information is available free of charge on the ACS Publications website at DOI:

Design of DNA sequences, materials, characterization and calibration experiments are in the Supporting Information File (PDF). Raw data files are also available at DOI: http://dx.doi.org/10.5258/SOTON/399859

\section{AUTHOR INFORMATION}

Corresponding author: *E-mail: A.Kanaras@ soton.ac.uk

Notes

The authors declare no competing financial interests.

\section{ACKNOWLEDGMENT}

PV would like to thank the University of Southampton and EPSRC for financial support via a DTA studentship. ATDBio is gratefully acknowledged for technical support regarding the oligonucleotide synthesis.

\section{REFERENCES}

(1) Contag, C. H.; Bachmann, M. H. Advances in in Vivo Bioluminescence Imaging of Gene Expression. Annu. Rev. Biomed. Eng. 2002, 4, 235-260.

(2) Golub, T. R.; Slonim, D. K.; Tamayo, P.; Huard, C.; Gaasenbeek, M.; Mesirov, J. P.; Coller, H.; Loh, M. L.; Downing, J. R.; Caligiuri, M. A.; Bloomfield, C. D.; Lander, E. S. Molecular Classification of Cancer: Class Discovery and Class Prediction by Gene Expression Monitoring. Science (80-. ). 1999, 286 (5439), 531-537.

(3) Beer, D. G.; Kardia, S. L. R.; Huang, C.-C.; Giordano, T. J.; Levin, A. M.; Misek, D. E.; Lin, L.; Chen, G.; Gharib, T. G.; Thomas, D. G.; Lizyness, M. L.; Kuick, R.; Hayasaka, S.; Taylor, J. M. G.; lannettoni, M. D.; Orringer, M. B.; Hanash, S. Gene-Expression Profiles Predict Survival of Patients
(4)

with Lung Adenocarcinoma. Nat. Med. 2002, 8 (8), 816824.

Chang, H. Y.; Sneddon, J. B.; Alizadeh, A. A.; Sood, R.; West, R. B.; Montgomery, K.; Chi, J. T.; Van De Rijn, M.; Botstein, D.; Brown, P. O. Gene Expression Signature of Fibroblast Serum Response Predicts Human Cancer Progression: Similarities between Tumors and Wounds. PLoS Biol. 2004, 2 (2). DOI: 10.1371/journal.pbio.0020007

(5) Hughes, J. P.; Rees, S. S.; Kalindjian, S. B.; Philpott, K. L. Principles of Early Drug Discovery. British Journal of Pharmacology. 2011, pp 1239-1249.

(6) Sahin, U.; Karikó, K.; Türeci, Ö. mRNA-Based Therapeutics--Developing a New Class of Drugs. Nat. Rev. Drug Discov. 2014, 13 (10), 759-780.

Pipinikas, C. P.; Carter, N. D.; Corbishley, C. M.; Fenske, C. D. HIF-1alpha mRNA Gene Expression Levels in Improved Diagnosis of Early Stages of Prostate Cancer. Biomarkers 2008, 13 (7), 680-691.

(8) Ng, C. F.; Yeung, R.; Chiu, P. K. F.; Lam, N. Y.; Chow, J.; Chan, B. The Role of Urine Prostate Cancer Antigen 3 mRNA Levels in the Diagnosis of Prostate Cancer among Hong Kong Chinese Patients. Hong Kong Med. J. 2012, 18 (6), 459-465.

(9) Perdonà, S.; Bruzzese, D.; Ferro, M.; Autorino, R.; Marino, A.; Mazzarella, C.; Perruolo, G.; Longo, M.; Spinelli, R.; Di Lorenzo, G.; Oliva, A.; De Sio, M.; Damiano, R.; Altieri, V.; Terracciano, D. Prostate Health Index (Phi) and Prostate Cancer Antigen 3 (PCA3) Significantly Improve Diagnostic Accuracy in Patients Undergoing Prostate Biopsy. Prostate 2013, 73 (3), 227-235.

(10) Friess, H.; Streit, S.; Michalski, C. W.; Erkan, M.; Kleeff, J. Northern Blot Analysis for Detection and Quantification of RNA in Pancreatic Cancer Cells and Tissues. Nat. Protoc. 2009, 4 (1), 37-43.

(11) Katagiri, F.; Glazebrook, J. Overview of mRNA Expression Profiling Using DNA Microarrays. Curr. Protoc. Mol. Biol. 2009, Chapter 22 (January), Unit 22.4.

Brewer, P. B.; Heisler, M. G.; Hejátko, J.; Friml, J.; Benková, E. In Situ Hybridization for mRNA Detection in Arabidopsis Tissue Sections. Nat. Protoc. 2006, 1 (3), 1462-1467.

Stylianopoulou, E.; Lykidis, D.; Ypsilantis, P.; Simopoulos, C.; Skavdis, G.; Grigoriou, M. A Rapid and Highly Sensitive Method of Non Radioactive Colorimetric in Situ Hybridization for the Detection of mRNA on Tissue Sections. PLoS One 2012, 7 (3). doi:10.1371/journal.pone.0033898.

(14) Wong, M. L.; Medrano, J. F. Real-Time PCR for mRNA Quantitation. BioTechniques. 2005, pp 75-85.

(15) Zhang, C.-Y.; Yeh, H.-C.; Kuroki, M. T.; Wang, T.-H. SingleQuantum-Dot-Based DNA Nanosensor. Nat. Mater. 2005, 4 (11), 826-831. 
Cady, N. C.; Strickland, A. D.; Batt, C. A. Optimized Linkage and Quenching Strategies for Quantum Dot Molecular Beacons. Mol. Cell. Probes 2007, 21 (2), 116124.

(17) Suzuki, M.; Husimi, Y.; Komatsu, H.; Suzuki, K.; Douglas, K. T. Quantum Dot FRET Biosensors That Respond to $\mathrm{pH}$, to Proteolytic or Nucleolytic Cleavage, to DNA Synthesis, or to a Multiplexing Combination. J. Am. Chem. Soc. 2008, 130 (17), 5720-5725.

(18) Lee, K.; Rouillard, J. M.; Kim, B. G.; Gulari, E.; Kim, J. Conjugated Polymers Combined with a Molecular Beacon for Label-Free and Self-Signal-Amplifying DNA Microarrays. Adv. Funct. Mater. 2009, 19 (20), 3317-3325.

(19) Gao, L.; Lian, C.; Zhou, Y.; Yan, L.; Li, Q.; Zhang, C.; Chen, L.; Chen, K. Graphene Oxide-DNA Based Sensors. Biosens. Bioelectron. 2014, 60, 22-29.

(20) Shi, J.; Tian, F.; Lyu, J.; Yang, M. Nanoparticle Based Fluorescence Resonance Energy Transfer (FRET) for Biosensing Applications. J. Mater. Chem. B 2015, 3 (35), 6989-7005.

(21) Smith, A. M.; Nie, S. Semiconductor Nanocrystals: Structure, Properties, and Band Gap Engineering. Acc. Chem. Res. 2010, 43 (2), 190-200.

(22) Haase, M.; Schäfer, H. Upconverting Nanoparticles. Angewandte Chemie - International Edition. 2011, pp 5808-5829.

(23) Xu, C. T.; Svenmarker, P.; Liu, H.; Wu, X.; Messing, M. E.; Wallenberg, L. R.; Andersson-Engels, S. High-Resolution Fluorescence Diffuse Optical Tomography Developed with Nonlinear Upconverting Nanoparticles. ACS Nano 2012, 6 (6), 4788-4795.

(24) Wang, Y. F.; Liu, G. Y.; Sun, L. D.; Xiao, J. W.; Zhou, J. C.; Yan, C. H.; Al, W. E. T. Nd 3 P -Sensitized Upconversion Nanophosphors : E Ffi Cient In Vivo Bioimaging Probes with Minimized Heating E Ff Ect. ACS Nano 2013, 7 (8), 7200-7206.

(25) Chen, F.; Bu, W.; Zhang, S.; Liu, J.; Fan, W.; Zhou, L.; Peng, W.; Shi, J. Gd3+Ion-Doped Upconversion Nanoprobes: Relaxivity Mechanism Probing and Sensitivity Optimization. Adv. Funct. Mater. 2013, 23 (3), 298-307.

(26) Liu, J.; Liu, Y.; Bu, W.; Bu, J.; Sun, Y.; Du, J.; Shi, J. Ultrasensitive Nanosensors Based on Upconversion Nanoparticles for Selective Hypoxia Imaging in Vivo upon near-Infrared Excitation. J. Am. Chem. Soc. 2014, $136(27), 9701-9709$.

(27) Lu, C. H.; Yang, H. H.; Zhu, C. L.; Chen, X.; Chen, G. N. A Graphene Platform for Sensing Biomolecules. Angew. Chemie - Int. Ed. 2009, 48 (26), 4785-4787.
Li, S.; Aphale, A. N.; MacWan, I. G.; Patra, P. K.; Gonzalez, W. G.; Miksovska, J.; Leblanc, R. M. Graphene Oxide as a Quencher for Fluorescent Assay of Amino Acids, Peptides, and Proteins. ACS Appl. Mater. Interfaces 2012, 4 (12), 7069-7075.

(29) Wu, M.; Kempaiah, R.; Huang, P. J. J.; Maheshwari, V.; Liu, J. Adsorption and Desorption of DNA on Graphene Oxide Studied by Fluorescently Labeled Oligonucleotides. Langmuir 2011, 27 (6), 2731-2738.

(30) Park, J. S.; Goo, N.; Kim, D. Supporting Information Mechanism of DNA Adsorption and Desorption on Graphene Oxide. Langmuir 2014, 30 (c), 1-3.

(31) Alonso-Cristobal, P.; Vilela, P.; El-Sagheer, A.; LopezCabarcos, E.; Brown, T.; Muskens, O. L.; Rubio-Retama, J.; Kanaras, a G. Highly Sensitive DNA Sensor Based on Upconversion Nanoparticles and Graphene Oxide. ACS Appl. Mater. Interfaces 2015, 7, 12422-12429.

(32) Querfurth, H. W.; Laferla, F. M. Alzheimer's Disease. N Engl J Med, 2010, 329-344.

De Kok, J. B.; Verhaegh, G. W.; Roelofs, R. W.; Hessels, D.; Kiemeney, L. A.; Aalders, T. W.; Swinkels, D. W.; Schalken, J. A. DD3PCA3, a Very Sensitive and Specific Marker to Detect Prostate Tumors. Cancer Res. 2002, 62 (9), 26952698.

(34) Marks, L. S.; Bostwick, D. G. Prostate Cancer Specificity of PCA3 Gene Testing: Examples from Clinical Practice. Rev. Urol. 2008, 10 (3), 175-181.

(35) Crawford, E. D.; Rove, K. O.; Trabulsi, E. J.; Qian, J.; Drewnowska, K. P.; Kaminetsky, J. C.; Huisman, T. K.; Bilowus, M. L.; Freedman, S. J.; Glover, W. L.; Bostwick, D. G. Diagnostic Performance of PCA3 to Detect Prostate Cancer in Men with Increased Prostate Specific Antigen: A Prospective Study of 1,962 Cases. J. Urol. 2012, 188 (5), 1726-1731.

(36) Qian, H.; Li, Z.; Zhang, Y. Multicolor Polystyrene Nanospheres Tagged with up-Conversion Fluorescent Nanocrystals. Nanotechnology 2008, 19 (25), 255601255604.

Liu, C.; Wang, H.; Li, X.; Chen, D. Monodisperse, SizeTunable and Highly Efficient $\beta-\mathrm{NaYF4:Yb,Er(Tm)} \mathrm{up-}$ Conversion Luminescent Nanospheres: Controllable Synthesis and Their Surface Modifications. J. Mater. Chem. 2009, 19 (21), 3546.

(38) Lin, W.; Fritzs, K.; Guerin, G.; Bardajee, G. R.; Hinds, S.; Sukhovatkin, V.; Sargent, E. H.; Scholes, G. D.; Winnik, M. A. Highly Luminescent Lead Sulfide Nanocrystals in Organic Solvents and Water through Ligand Exchange with Poly(acrylic Acid). Langmuir 2008, 24 (15), 8215 8219. 


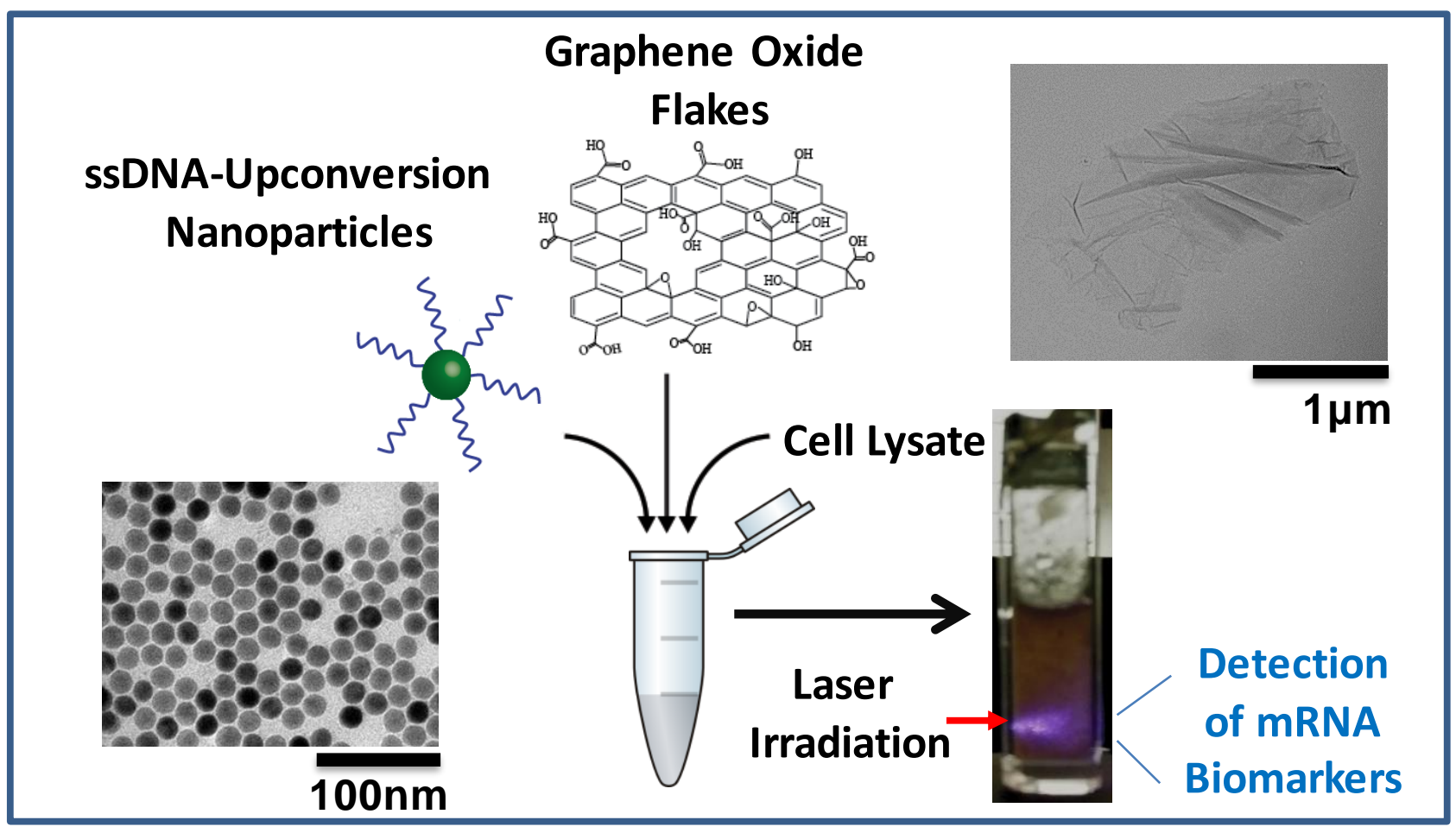

